\title{
The photon structure function measurements from LEP
}

\author{
Richard Nisius, on behalf of the LEP Collaborations * \\ PPE Division, CERN, CH-1211 Genève 23, Switzerland
}

October 7, 2018

\begin{abstract}
The present knowledge of the structure of the photon based on measurements of photon structure functions is discussed. This review covers QED structure functions and the hadronic structure function $F_{2}^{\gamma}$.
\end{abstract}

\section{Introduction}

One of the most powerful tools to investigate the internal structure of quasi real photons is the measurement of photon structure functions in deep inelastic electron photon scattering at $\mathrm{e}^{+} \mathrm{e}^{-}$colliders. These measurements have by now a tradition of sixteen years since the first $F_{2}^{\gamma}$ was obtained by PLUTO [1]. The LEP accelerator is a unique place for the measurements of photon structure functions until a high energy linear collider is realised. It is unique because of the large coverage in $Q^{2}$ owing to the various beam energies covered within the LEP programme, and due to the high luminosities delivered to the experiments. Structure function measurements are performed by all four LEP experiments, however concentrating on different aspects of the photon structure. The main idea is that by measuring the differential cross section

$$
\frac{d^{2} \sigma_{e \gamma \rightarrow e X}}{d x d Q^{2}}=\frac{2 \pi \alpha^{2}}{x Q^{4}}\left[\left(1+(1-y)^{2}\right) F_{2}^{\gamma}\left(x, Q^{2}\right)-y^{2} F_{\mathrm{L}}^{\gamma}\left(x, Q^{2}\right)\right]
$$

one obtains the photon structure function $F_{2}^{\gamma}$ which is proportional to the parton content of the photon and therefore reveals the internal structure of the photon. Here $Q^{2}$ is the absolute value of the four momentum squared of the virtual photon, $x$ and $y$ are the usual dimensionless variables of deep inelastic scattering and $\alpha$ is the fine structure constant. In the region of small $y$ studied $(y \ll 1)$ the contribution of the term proportional to $F_{\mathrm{L}}^{\gamma}\left(x, Q^{2}\right)$ is small and it is usually neglected.

\footnotetext{
*Invited talk given at the International Europhysics Conference on High Energy Physics, 19-26 August 1997, Jerusalem, Israel, to appear in the Proceedings.
} 
Because the energy of the quasi-real photon is not known, $x$ has to be derived by measuring the invariant mass of the final state $X$, which consists of $\mu^{+} \mu^{-}$ pairs for $F_{\mathrm{k}, \mathrm{QED}}^{\gamma}, k=2, A, B$, and of hadrons created by a q $\overline{\mathrm{q}}$ pair in studies of $F_{2}^{\gamma}$. The invariant mass can be determined accurately in the case of $\mu^{+} \mu^{-}$final states, and measurements of $F_{\mathrm{k}, \mathrm{QED}}^{\gamma}$ are statistically limited. For hadronic final states the measurement of $x$ is a source of significant uncertainties which makes measurements of $F_{2}^{\gamma}$ mainly systematics limited.

\section{The QED structure functions}

Several measurements of QED structure functions from LEP have been submitted to this conference. The $\mu^{+} \mu^{-}$final state is such a clean environment that it allows for much more subtle measurements to be performed than in the case of hadronic final states. Therefore the investigation of QED structure functions serves not only as a test of QED but rather it is used to refine the experimentalists tools in a real but clean environment to investigate the possibilities of extracting similar information from the much more complex hadronic final states.

$\chi$ is defined as the azimuthal angle between the plane spanned by the muon pair and the plane spanned by the incoming quasi-real photon and the deep inelastically scattered electron. This angle has been used to extract structure functions of the photon, $F_{\mathrm{A}, \mathrm{QED}}^{\gamma}$ and $F_{\mathrm{B}, \mathrm{QED}}^{\gamma}$, which can not be assessed by the cross section measurement, which is dominated by the contribution of $F_{2}^{\gamma}$ QED Azimuthal correlations based on $\chi$ were investigated by ALEPH (prel.) [2], L3 (prel.) [3] and OPAL [4], and $F_{2, \text { QED }}^{\gamma}$ has been obtained by all four experiments. Figure 11 shows the world summary of the $F_{2, \mathrm{QED}}^{\gamma}$ measurements [2, 3, 5] 9]. All measurements are consistent with expectations from QED. The LEP data are so precise that it is possible to study the effect of the small virtuality $P^{2}$ of the quasi real photon 3,6 .

\section{The hadronic structure function $F_{2}^{\gamma}$}

The measurement of $F_{2}^{\gamma}$ has attracted a lot of interest at LEP over the last years. The LEP Collaborations have measured $F_{2}^{\gamma}$ in the range $0.0025<x \lesssim$ 1 and $\left(1.86<Q^{2}<279\right) \mathrm{GeV}^{2}$, the largest range ever studied 10 14. This work has also encountered some difficulties [10,14] which were not considered in older determinations of $F_{2}^{\gamma}$. Two distinct features of the photon structure are investigated. Firstly the shape of $F_{2}^{\gamma}$ is measured as a function of $x$ at fixed $Q^{2}$. Particular emphasis is put on measuring the low- $x$ behaviour of $F_{2}^{\gamma}$ in comparison to $F_{2}^{\mathrm{p}}$ as obtained at HERA. Secondly the evolution of $F_{2}^{\gamma}$ with $Q^{2}$ at medium $x$ is investigated. This evolution is predicted by QCD to be logarithmic. In general $F_{2}^{\gamma}$ is found to rise smoothly towards large $x$ and there 
is some weak indication for a possible rise at low $x$ for $Q^{2}<4 \mathrm{GeV}^{2}$, as shown in Fig 2. This behaviour is satisfactorily described (except in Fig 2b) by several of the existing $F_{2}^{\gamma}$ parametrisation, e.g. GRV [15, 16] and SaS [17]. Experimentally there seems to emerge an inconsistency between the OPAL [12] and PLUTO [18] data on one hand and the TPC [19] data on the other hand at $Q^{2} \approx 4 \mathrm{GeV}^{2}$, see $[12$.

The evolution of $F_{2}^{\gamma}$ with $Q^{2}$, Fig 3, has been studied using the large lever arm in $Q^{2}$, and also by comparing various ranges in $x$ within one experiment [11]. Unfortunately the different experiments quote their results for different ranges in $x$ which makes the comparison more difficult because the predictions for the various ranges in $x$ start to be significantly different for $Q^{2}>100 \mathrm{GeV}^{2}$, as can be seen in Fig 3. The measurements are consistent with each other and a clear rise of $F_{2}^{\gamma}$ with $Q^{2}$ is observed. It is an interesting fact that this rise can be described reasonably well $(\mathcal{O}(15 \%)$ accuracy) by the leading order asymptotic solution [20] for $F_{2}^{\gamma}$ as predicted by perturbative QCD for $\alpha_{s}\left(M_{z}^{2}\right)=0.128$ as detailed in [1].

\section{Conclusion}

New informations on the photon structure in so far unexplored $Q^{2}$ and $x$ regions have been obtained based on structure function measurements at LEP. All observations are consistent with the QED and QCD predictions. The future LEP2 programme will allow to extend the study of the photon structure up to $Q^{2} \approx 1000 \mathrm{GeV}^{2}$.

Acknowledgement: I wish to thank the members of the LEP collaborations for their help during the preparation of this review.

\section{References}

[1] PLUTO Collab., C. Berger et al., Phys. Lett. 107B, 168-172 (1981).

[2] ALEPH Collab., contributed paper to Photon 97, Egmond aan Zee.

[3] L3 Collab., contributed paper to this conference.

[4] OPAL Collab., K. Ackerstaff et al., Z. Phys. C74, 49-55 (1997).

[5] OPAL Collab., R. Akers et al., Z. Phys. C60, 593-600 (1993).

[6] DELPHI Collab., P. Abreu et al., Z. Phys. C69, 223-234 (1996).

[7] TPC/2 $\gamma$ Collab., M. P. Cain et al., Phys. Lett. 147B, 232-236 (1984).

[8] CELLO Collab., H. J. Behrend et al., Phys. Lett. 126B, 384-390 (1983). 
[9] PLUTO Collab., C. Berger et al., Z. Phys. C27, 249-256 (1985).

[10] OPAL Collab., K. Ackerstaff et al., Z. Phys. C74, 33-48 (1997).

[11] OPAL Collab., K. Ackerstaff et al., Phys. Lett. B411, 387-401 (1997).

[12] OPAL Collab., K. Ackerstaff et al., Phys. Lett. B412, 225-234 (1997).

[13] DELPHI Collab., Proceedings of Photon 97, Egmond aan Zee.

[14] ALEPH Collab., contributed paper to this conference.

[15] M. Glück, E. Reya, and A. Vogt, Phys. Rev. D45, 3986-3994 (1992).

[16] M. Glück, E. Reya, and A. Vogt, Phys. Rev. D46, 1973-1979 (1992).

[17] G. A. Schuler and T. Sjöstrand, Z. Phys. C68, 607-623 (1995).

[18] PLUTO Collab., C. Berger et al., Phys. Lett. 142B, 111-118 (1984).

[19] TPC/2 $\gamma$ Collab., H. Aihara et al., Z. Phys. C34, 1-13 (1987).

[20] E. Witten, Nucl. Phys. B120, 189-202 (1977). 


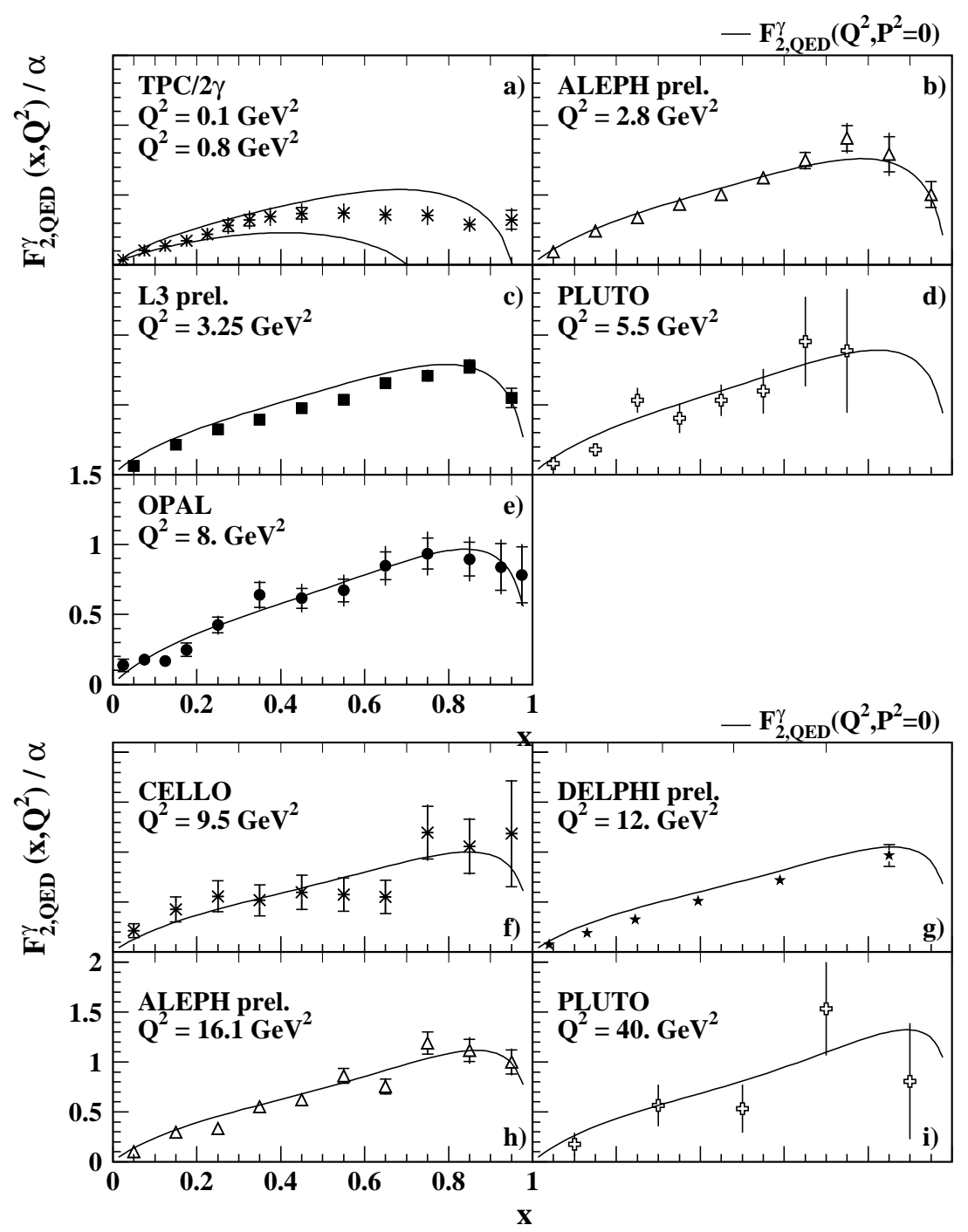

Figure 1: The measurements of $F_{2, \mathrm{QED}}^{\gamma}$ as a function of $x$ for various $Q^{2}$ compared to $Q E D$ assuming $P^{2}=0$. The $Q^{2}$ values for the predictions are taken from the publications. If the data were unfolded to a given $Q^{2}$ this value is taken, if only the average $Q^{2}$ of the sample is given, c), this value is taken, and if no value at all is quoted the $Q^{2}$ range as obtained from the information of the event selection is shown, a). The quoted errors for g) are statistical only. 

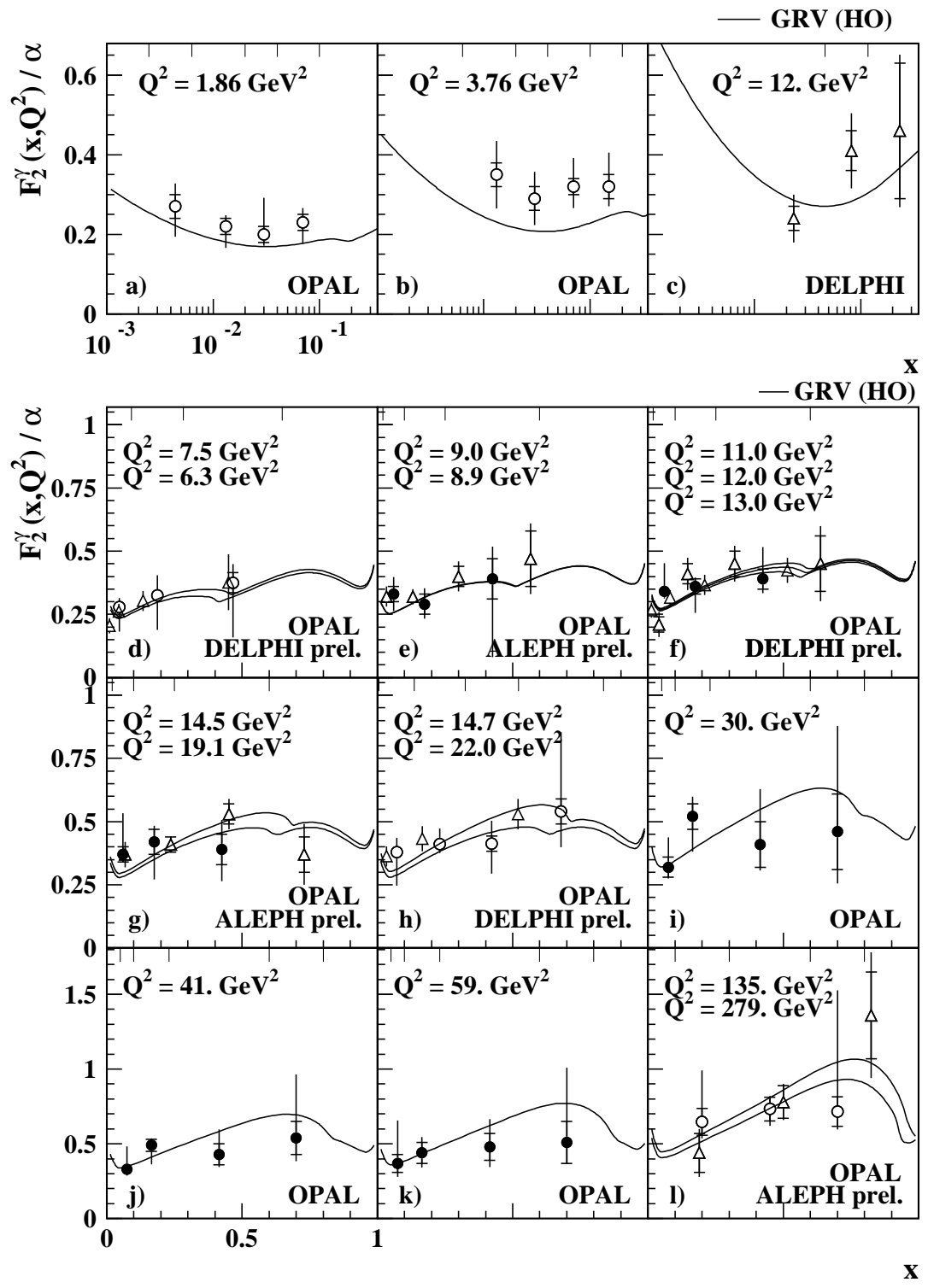

Figure 2: The measurements of $F_{2}^{\gamma}$ as a function of $x$ unfolded on a logarithmic $x$ scale, $a)-c)$, or on a linear $x$ scale, $d)-l)$, compared to the prediction of the GRV (HO) parametrisation. The OPAL data at $11 \mathrm{GeV}^{2}\left(41 \mathrm{GeV}^{2}\right)$ are the combined data from 9 and $14.5 \mathrm{GeV}^{2}$ (30 and $59 \mathrm{GeV}^{2}$ ). The inner error bar is the statistical error and the outer the quadratic sum of statistical and systematic error. (ALEPH, DELPHI triangles, OPAL circles) 


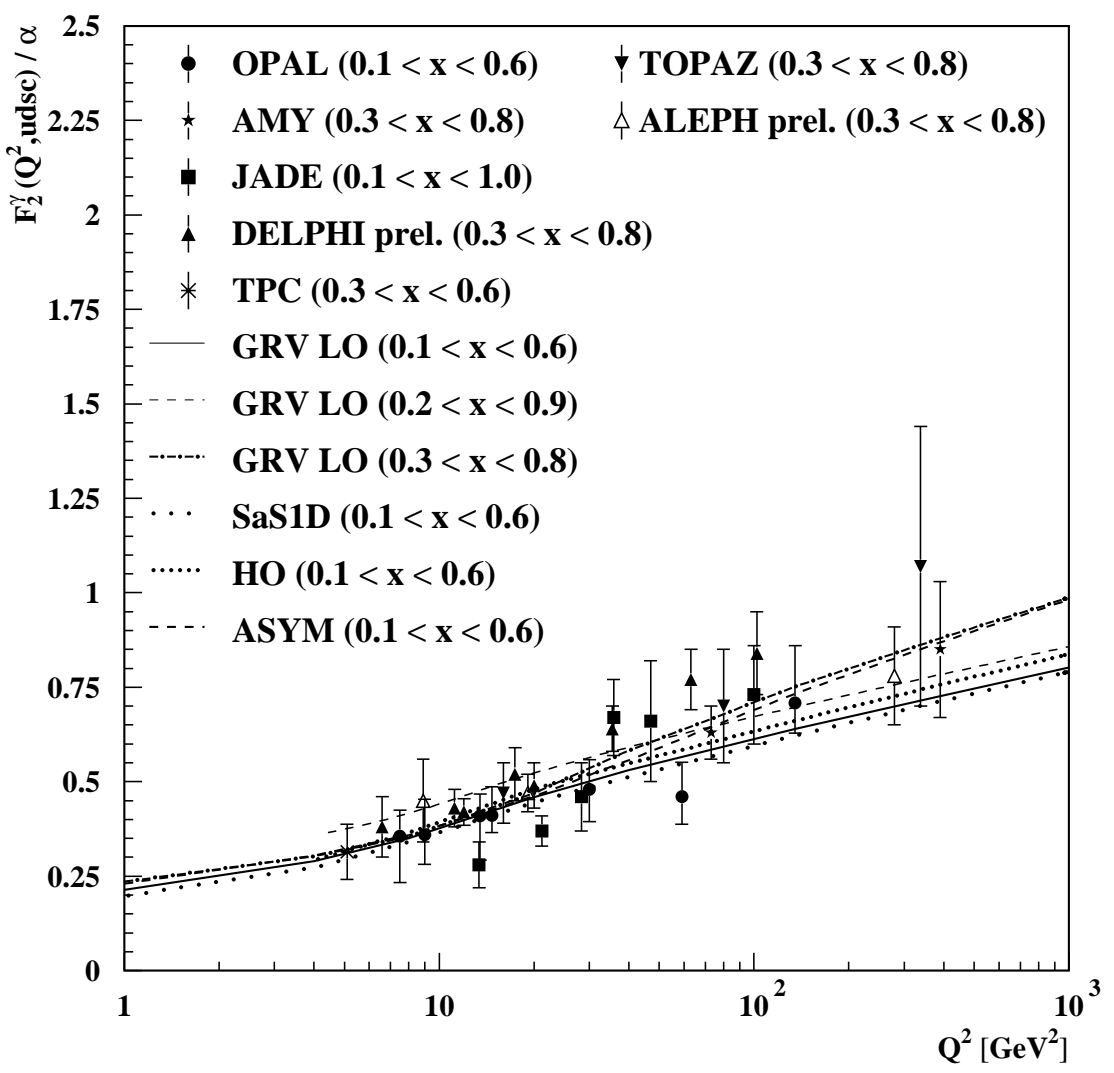

Figure 3: Summary of the measurements of $F_{2}^{\gamma}$ at medium $x$ without subtraction of the charm contribution compared to several theoretical predictions taking into account the various ranges in $x$. 